AperTO - Archivio Istituzionale Open Access dell'Università di Torino

\title{
A characterization for solutions of stochastic discrete time optimization models
}

\section{This is the author's manuscript}

Original Citation:

Availability:

This version is available http://hdl.handle.net/2318/135697

since

Published version:

DOI:10.1007/BF02096426

Terms of use:

Open Access

Anyone can freely access the full text of works made available as "Open Access". Works made available under a Creative Commons license can be used according to the terms and conditions of said license. Use of all other works requires consent of the right holder (author or publisher) if not exempted from copyright protection by the applicable law. 


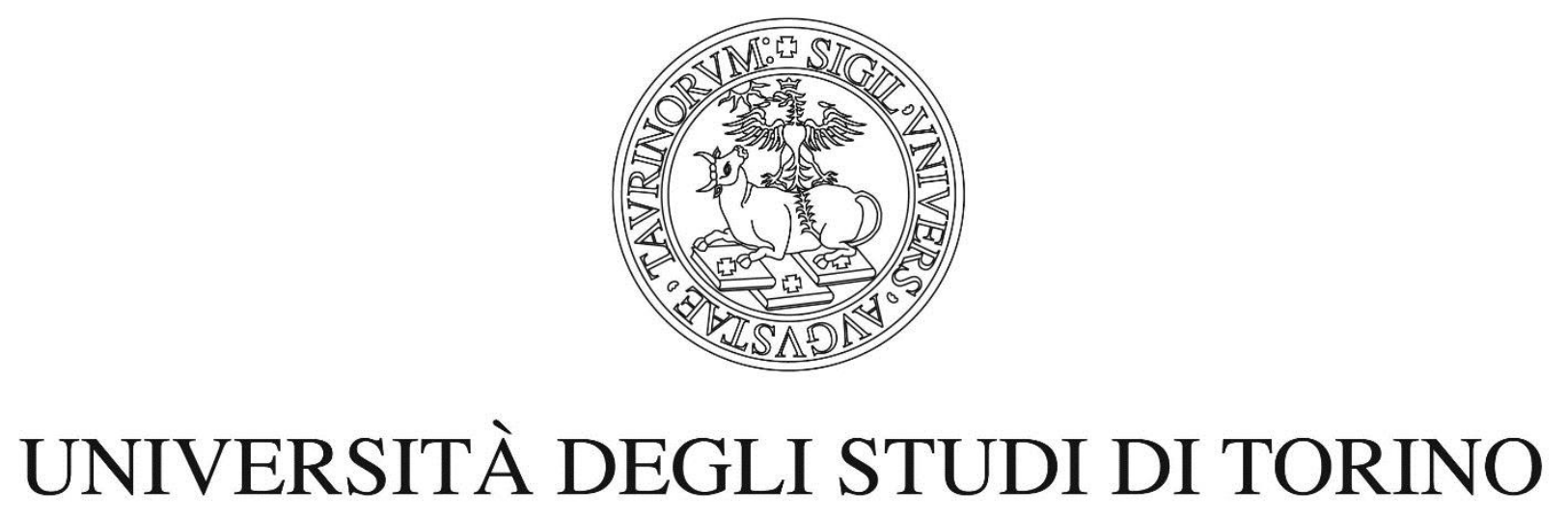

This is an author version of the contribution published on:

Questa è la versione dell'autore dell'opera:

Privileggi, F. (1995), A Characterization for Solutions of Stochastic Discrete Time Optimization Models, Rivista di Matematica per le Scienze Economiche e Sociali Vol.

18, Issue 2: pp. 165-180, DOI 10.1007/BF02096426

The definitive version is available at:

La versione definitiva è disponibile alla URL:

http://link.springer.com/article/10.1007\%2FBF02096426 


\title{
A Characterization for Solutions of Stochastic Discrete Time Optimization Models
}

\author{
Fabio Privileggi \\ Dipartimento di Matematica Applicata "Bruno de Finetti" \\ Università di Trieste \\ P.le Europa 1, 34127 - Trieste \\ Tel. +39-40-6767117; Fax +39-40-567136 \\ E-mail privileg@univ.trieste.it
}

Draft: December 1995

\begin{abstract}
A notion of finitely optimal plan for intertemporal optimization problems as a necessary condition for optimality is introduced. Under interiority of a feasible plan and differentiability of the return function, such a plan satisfies the stochastic analogue of deterministic Euler-Lagrange conditions, which become also sufficient conditions under concavity of the return function. Then, under more general assumptions, a sufficient criterion of optimality based on competitive plans supported by price systems and transversality conditions is discussed. Differently from the current literature, no restrictive hypotheses on the probability measure of the random shocks are assumed.
\end{abstract}

\section{Introduction}

Ever since dynamic programming techniques have been introduced to solve intertemporal optimization problems under uncertainty, a parallel approach based on the calculus of variations has been developing to characterize their solutions. In many economic problems optimality conditions for feasible plans are stated in terms of the stochastic version of Euler-Lagrange conditions (primarily in the optimal growth literature; see [6], [12], [17], [9], [2] and, more recently, [7], [16]); in all these papers such conditions are defined specifically to prove main results for the model to discuss. Only in [1] the problem is treated in a more general perspective, however under the standard and rather restrictive assumptions adopted in dynamic programming; that is, under differentiability, monotonicity and concavity of the return function and assuming that the exogenous shocks are described by a first order Markov process with a dense stationary transition function.

The aim of this paper is to build up a simple but powerful method to identify optimal plans giving sufficient conditions of optimality under very general hypotheses, particularly with regard to the stochastic process of the exogenous shocks. Firstly we present the first order necessary conditions for optimal plans stated in terms of stochastic Euler-Lagrange equations under interiority of the plan and differentiability of the return function. Furthermore we show that, if the return function is concave, these conditions are also sufficient for a weaker criterion 
of optimality, called finite optimality. Secondly we look at the more general case in which the return function is only superdifferentiable and state the competitivity criterion for feasible plans: we will say that a plan is competitive if it is supported by a sequence of random variables, each interpretable as a random shadow price. This criterion gives sufficient conditions for finite optimality without concavity assumptions on the return function; furthermore, adding a transversality condition, these conditions are sufficient also for optimality (that is not only for finite optimality). Competitive plans in a stochastic environment are already discussed in a recent article (see [13]), where a different approach has been pursued: under the standard assumptions of the optimal growth model, plans supported by a random price system are defined as necessary conditions, following a methodology similar to that developed in [8] for the deterministic case. Here an easily handled method, based on sufficient conditions, is presented ${ }^{1}$.

We stress that, unlike the current stream of research, we do not impose any kind of restriction upon the stochastic process representing the exogenous shocks: it is a process characterized by any probability measure. The key argument adopted in most of the proofs is due to Ionescu Tulcea's canonical procedure to build a stochastic process (see chapter V in [11]): any stochastic process can be generated by a sequence of stochastic kernels, each of them representing the probability measure at time $t$ conditioned to the story of shocks occurred until time $t-1$.

\section{The Infinite Horizon Optimization Problem}

In this section we set up the stochastic intertemporal optimization problem giving assumptions under which it is well defined (see chapter 9 in [14] for a more detailed description). In addition we state, without proof, the Ionescu Tulcea's Proposition and an important Corollary that determines the additive separability of the expected total return function. This will be used later in the proofs of the main results.

A discrete time infinite horizon optimization problem under uncertainty is characterized by the tuple $\left(X, Z, \Gamma, F, \beta,\left\{Q_{t}\right\}\right)$.

(i) $X \subseteq \mathbf{R}^{l}$ is the (Borel-measurable) state space for the system and the action space for the decision maker as well; let $\mathcal{X} \subseteq \mathcal{B}^{l}$ be the Borel $\sigma$-algebra on $X$.

(ii) $Z \subseteq \mathbf{R}^{k}$ is the random events space describing the exogenous shocks affecting the system; let $\mathcal{Z} \subseteq \mathcal{B}^{k}$ be the Borel $\sigma$-algebra on $Z$.

(iii) $\Gamma: X \times Z \rightarrow X$ is a correspondence representing the one-period constraint; that is, $\Gamma(x, z)$ is the set of feasible values for next period's endogenous state variable if the current state is $(x, z)$. Let $A=\{(x, y, z) \in X \times X \times Z: y \in \Gamma(x, z)\}$ be the graph of $\Gamma$.

(iv) The one-period return function $F: A \rightarrow \mathbf{R}$ is the immediate reward; that is, $F(x, y, z)$ is the current-period reward to the decision maker if action $y \in \Gamma(x, z)$ is chosen when the current state is $(x, z)$.

(v) $\beta \geq 0$ is the (constant) one-period discount factor.

(vi) $\left\{Q_{t}\right\}$ is a sequence of stochastic kernels which describe the evolution law of the random shocks. For each $t, Q_{t}: Z^{t} \times \mathcal{Z} \rightarrow[0,1]$ is a function measurable with respect to the first $t-1$ variables and a probability measure with respect to the last variable; it represents the probability measure of the random shocks at time $t$ given the previous story of shocks

\footnotetext{
${ }^{1} \mathrm{~A}$ similar approach for the deterministic case can be found in [10].
} 
$\left(z_{1}, \ldots, z_{t-1}\right)$ occurred until time $t-1$ : that is ${ }^{2}, Q_{t}\left(z_{0}, \ldots, z_{t-1} ; B\right)=\operatorname{Pr}\left(z_{t} \in B \mid z_{0}, \ldots, z_{t-1}\right)$, all $B \in \mathcal{Z}$.

The objective of the decision maker is to choose a sequence of actions which will maximize the expected discounted total reward subject to his constraints. Given the measurable spaces $(X, \mathcal{X})$ and $(Z, \mathcal{Z})$, let $(S, \mathcal{S})=(X \times Z, \mathcal{X} \otimes \mathcal{Z})$ the product space representing the state of the system, where $s_{t}=\left(x_{t}, z_{t}\right)$ is an element of the state space at date $t$. Now we need to be more precise with what we mean by sequences of actions, the set over which the maximization occurs, and which is the probability measure of the expected total reward. Since it is unknown at the initial time $t=0$ which action will be chosen at any future date, because it is unknown which sequence of shocks will affect the system until that date, feasible plans must be defined in terms of sequences of random variables. Fix the initial state $s_{0}=\left(x_{0}, z_{0}\right) \in S$ and let $z^{t}=\left(z_{1}, \ldots, z_{t}\right) \in Z^{t}=Z \times \ldots \times Z$ ( $t$ times) a partial story of shocks from period 1 to period $t$.

Definition $1 A$ plan $\pi$ is a value $\pi_{0} \in X$ and a sequence $\left\{\pi_{t}\right\}$ of measurable functions $\pi_{t}$ : $Z^{t} \rightarrow X, t=1,2, \ldots$ A plan $\pi$ is feasible from $s_{0} \in S$ if

$$
\begin{aligned}
& \pi_{0} \in \Gamma\left(s_{0}\right) \text { and } \\
& \pi_{t}\left(z^{t}\right) \in \Gamma\left[\pi_{t-1}\left(z^{t-1}\right), z_{t}\right], \text { a.e., } t=1,2, \ldots .
\end{aligned}
$$

Let $\Pi\left(s_{0}\right)$ denote the set of plans that are feasible from $s_{0}$.

A. $1 \Pi\left(s_{0}\right)$ is nonempty for all $s_{0} \in S$.

A sufficient condition is $X$ to be closed and $\Gamma: S \rightarrow X$ to be non-empty valued, closed and upper semi-continuous. Under this condition, Proposition 1, p.22, and Lemma 1, p. 55, in [4], ensure the existence of a measurable selection from $\Gamma$; that is, there exists a measurable function $h: S \rightarrow X$ such that $h(s) \in \Gamma(s)$, all $s \in S$. Thus, for all $s_{0} \in S$, one can define a plan $\pi$ recursively ${ }^{3}$ by $\pi_{0}=h\left(s_{0}\right)$ and $\pi_{t}\left(z^{t}\right)=h\left[\pi_{t-1}\left(z^{t-1}\right), z_{t}\right]$, all $z^{t} \in Z^{t}, t=1,2, \ldots$

Next step is to define the probability measure of the stochastic process representing the exogenous shocks. Let $Z^{t}$ be the Cartesian product $Z \times \ldots \times Z(t$ times $)$ and $\mathcal{Z}^{t}$ be the product $\sigma$-algebra $\mathcal{Z} \otimes \ldots \otimes \mathcal{Z}$. Similarly let $\left(Z^{\infty}, \mathcal{Z}^{\infty}\right)$ denote the product space as the horizon recedes to infinity.

Proposition 1 (Ionescu Tulcea) Given a probability space $(Z, \mathcal{Z})$ and a sequence $\left\{Q_{t}\right\}$ of stochastic kernels $Q_{t}: Z^{t} \times \mathcal{Z} \rightarrow[0,1]$, for all $z_{0} \in Z$ there exists a unique probability measure $P\left(z_{0}, \cdot\right)$ on $\left(Z^{\infty}, \mathcal{Z}^{\infty}\right)$ whose value for every finite measurable rectangle $C=A_{1} \times A_{2} \times \ldots \times A_{t} \times$ $Z \times Z \times \ldots$, with $A_{i} \in \mathcal{Z}, 1 \leq i \leq t$, is given by

$$
\begin{aligned}
P\left(z_{0}, C\right) & =\lambda^{t}\left(z_{0}, C\right) \\
& =\int_{A_{1}} Q_{1}\left(z_{0} ; d z_{1}\right) \int_{A_{2}} Q_{2}\left(z_{0}, z_{1} ; d z_{2}\right) \ldots \int_{A_{t}} Q_{t}\left(z_{0}, \ldots, z_{t-1} ; d z_{t}\right),
\end{aligned}
$$

where $\lambda^{t}\left(z_{0}, \cdot\right)$ is a probability measure on the finite product space $\left(Z^{t}, \mathcal{Z}^{t}\right)$.

Since, given any probability measure $P$ for a stochastic process defined on $\left(Z^{\infty}, \mathcal{Z}^{\infty}\right)$, all the probability measures on finite-dimensional subspaces are uniquely determined, by Proposition 1 , to take the sequence of stochastic kernels $\left\{Q_{t}\right\}$ as a given of the problem is equivalent to give directly the probability $P$. Since $P$ is arbitrary, this means that we put no restrictions on the stochastic process of the random shocks.

\footnotetext{
${ }^{2}$ For simplicity, we use the same symbol for the random variable and the generic value it can assume.

${ }^{3}$ If we choose the same measurable selection $h$ in every period $t$, the plan is said to be stationary or Markov, since the action to be taken for each period $t$ depends only on the state $s_{t}=\left[\pi_{t-1}\left(z^{t-1}\right), z_{t}\right]$ of the system in that period. Nonstationary plans can be constructed by using different measurable selections $h_{t}$ in each period.
} 
Corollary 1 Given $(Z, \mathcal{Z}),\left\{Q_{t}\right\},\left(Z^{t}, \mathcal{Z}^{t}\right)$ and $\lambda^{t}\left(z_{0}, \cdot\right)$ defined above, if, for fixed $z_{0} \in Z$ and $t$, the function $f: Z^{t} \rightarrow \mathbf{R}$ is $\lambda^{t}\left(z_{0}, \cdot\right)$-integrable, then

$$
\int_{Z_{t}} f\left(z^{t}\right) \lambda^{t}\left(z_{0}, d z^{t}\right)=\int_{Z} Q_{1}\left(z_{0} ; d z_{1}\right) \int_{Z} Q_{2}\left(z_{0}, z_{1} ; d z_{2}\right) \ldots \int_{Z} Q_{t}\left(z_{0}, \ldots, z_{t-1} ; d z_{t}\right) f\left(z^{t}\right) .
$$

This corollary will be useful later, in most of the proofs, to brake up the total return function defined as the sum of expected rewards at each period. For a proof of Proposition 1 and Corollary 1 see Proposition V.1.1., p. 162, in [11].

To define the expected total return function, the one-period return function must be integrable, as next assumption states. Let $\mathcal{A}=\{C \in \mathcal{X} \otimes \mathcal{X} \otimes \mathcal{Z}: C \subseteq A\}$.

A. $2 F$ is $\mathcal{A}$-measurable and, for all $s_{0} \in S$ and all $\pi \in \Pi\left(s_{0}\right), F\left[\pi_{t-1}\left(z^{t-1}\right), \pi_{t}\left(z^{t}\right), z_{t}\right]$ is $\lambda^{t}\left(z_{0}, \cdot\right)$-integrable, $t=1,2, \ldots$.

Definition 2 Given $s_{0} \in S$ and a plan $\pi \in \Pi\left(s_{0}\right)$, the expected discounted total return is

$$
u\left(s_{0}, \pi\right)=F\left(x_{0}, \pi_{0}, z_{0}\right)+\liminf _{n \rightarrow \infty} \sum_{t=1}^{n} \beta^{t} \int_{Z^{t}} F\left[\pi_{t-1}\left(z^{t-1}\right), \pi_{t}\left(z^{t}\right), z_{t}\right] \lambda^{t}\left(z_{0}, d z^{t}\right) .
$$

Notice that choosing the "liminf" criterion allows us to include cases where the series of the expected discounted one-period returns may oscillate.

Definition 3 The value function of the optimization problem is

$$
v\left(s_{0}\right)=\sup _{\pi \in \Pi\left(s_{0}\right)} u\left(s_{0}, \pi\right) .
$$

In other words, the value function represents the maximum feasible total return to the decision maker. Finally we introduce two criterion of optimality for feasible plans which will be used in subsequent sections. Define the partial sums of the expected one-period returns as follows:

$$
\begin{aligned}
& u_{0}\left(s_{0}, \pi\right)=F\left(x_{0}, \pi_{0}, z_{0}\right) \text { and } \\
& u_{n}\left(s_{0}, \pi\right)=u_{0}\left(s_{0}, \pi\right)+\sum_{t=1}^{n} \beta^{t} \int_{Z^{t}} F\left[\pi_{t-1}\left(z^{t-1}\right), \pi_{t}\left(z^{t}\right), z_{t}\right] \lambda^{t}\left(z_{0}, d z^{t}\right), n=1,2, \ldots
\end{aligned}
$$

Definition 4 A feasible plan $\pi^{*} \in \Pi\left(s_{0}\right)$ is finitely optimal if, for any $n \geq 1$ and for any feasible plan $\pi \in \Pi\left(s_{0}\right)$ such that $\pi_{n}^{*}=\pi_{n}$ a.e., $u_{n}\left(s_{0}, \pi^{*}\right) \geq u_{n}\left(s_{0}, \pi\right)$ holds.

Definition 5 A feasible plan $\pi^{*} \in \Pi\left(s_{0}\right)$ is optimal if $v\left(s_{0}\right)=u\left(s_{0}, \pi^{*}\right)$ and $-\infty<v\left(s_{0}\right)<+\infty$.

\section{Characterization of Finitely Optimal Plans}

Some necessary conditions for a finitely optimal plan are presented in this section. First we show that the definition of finite optimality is itself a necessary condition for optimality. A second step is to characterize finitely optimal plans by an inequality that must be satisfied at any date $t$. Then we introduce an interiority notion for the plan and assume the differentiability of the one-period return function in order to define necessary conditions analogue to the well known Euler-Lagrange equations usually stated in the deterministic context. Finally we prove that if $F$ is concave these conditions become also sufficient for a finitely optimal plan. 
As we already pointed out in the introduction, Euler-Lagrange equations are frequently adopted in stochastic dynamic programming to give necessary conditions for optimality in both finite or infinite horizon problems. They are usually obtained as the first order condition applied to the Bellman functional equation, a procedure allowed by the envelope theorem that guarantees the differentiability of the value function under concavity and differentiability of the one-period return function. In this case the stochastic process of the exogenous shocks must be assumed to be a first order stationary Markov process in order to let the Bellman equation be defined. In our analysis no functional equations are introduced and the value function is not involved in computations; necessary conditions come out directly from the sequential problem as stated in definition 3, i.e. from the expected discounted total return function (which, in the differentiable case, doesn't need concavity assumptions). Since this approach appears to be new for the stochastic case, all the results of this section are given with proofs, which essentially are an application of Corollary 1 to Ionescu Tulcea Theorem. In this context, problems where uncertainty is described by a first order stationary Markov process can be viewed as a particular case in which the sequence of stochastic kernels $\left\{Q_{t}\right\}$ consists of the constant transition function $Q: Z \times \mathcal{Z} \rightarrow[0,1]$

Since, for every $t$, the random variables $\pi_{t}$ are defined on $Z^{t}$, from now on we will drop the argument for simplicity of notation; that is we will write $\pi_{t}$ instead of $\pi_{t}\left(z^{t}\right)$.

Proposition 2 If $\pi^{*} \in \Pi\left(s_{0}\right)$ is optimal, then is finitely optimal.

Proof. Since $\left(s_{0}, \pi^{*}\right)$ is optimal, $v\left(s_{0}\right)=u\left(s_{0}, \pi^{*}\right) \geq u\left(s_{0}, \pi\right)$ holds for all $\pi \in \Pi\left(s_{0}\right)$. Now, to prove finite optimality, suppose the contrary: assume that a plan $\pi \in \Pi\left(s_{0}\right)$ such that, for a certain $n \geq 1, \pi_{t}^{*}=\pi_{t}$ a.e. if $t \geq n$ and $u_{n}\left(s_{0}, \pi\right)>u_{n}\left(s_{0}, \pi^{*}\right)$, exists. Hence,

$$
\begin{aligned}
u_{n}\left(s_{0}, \pi\right)+\lim \inf _{k \rightarrow \infty} \sum_{t=n+1}^{k} \beta^{t} \int_{Z^{t}} F\left(\pi_{t-1}, \pi_{t}, z_{t}\right) \lambda^{t}\left(z_{0}, d z^{t}\right) & \\
& >u_{n}\left(s_{0}, \pi^{*}\right)+\lim \inf _{k \rightarrow \infty} \sum_{t=n+1}^{k} \beta^{t} \int_{Z^{t}} F\left(\pi_{t-1}, \pi_{t}, z_{t}\right) \lambda^{t}\left(z_{0}, d z^{t}\right),
\end{aligned}
$$

that is, $u\left(s_{0}, \pi\right)>u\left(s_{0}, \pi^{*}\right)$, which is a contradiction.

Finite optimality is necessary for optimality but, in general, is not sufficient; in order to reverse the implication, as we will see, we need to add a transversality condition. Now we state a more general necessary condition.

Proposition 3 If a plan $\pi^{*} \in \Pi\left(s_{0}\right)$ is optimal (or at least finitely optimal), then it satisfies a.e.

$$
\begin{aligned}
& F\left(\pi_{t-1}^{*}, y, z_{t}\right)+\beta \int_{Z} F\left(y, \pi_{t+1}^{*}, z_{t+1}\right) Q_{t+1}\left(z_{0}, \ldots, z_{t} ; d z_{t+1}\right) \\
& \quad \leq F\left(\pi_{t-1}^{*}, \pi_{t}^{*}, z_{t}\right)+\beta \int_{Z} F\left(\pi_{t}^{*}, \pi_{t+1}^{*}, z_{t+1}\right) Q_{t+1}\left(z_{0}, \ldots, z_{t} ; d z_{t+1}\right) \\
& \text { s.t. } y \in \Gamma\left(\pi_{t-1}^{*}, z_{t}\right) \text { and } \pi_{t+1}^{*} \in \Gamma\left(y, z_{t+1}\right), t=0,1, \ldots,
\end{aligned}
$$

where, for $t=0$, we agree to put $\pi_{t-1}^{*} \equiv x_{0}$.

Therefore, for each $t, \pi_{t}^{*}$ maximizes the function

$$
F\left(\pi_{t-1}^{*}, \cdot, z_{t}\right)+\beta \int_{Z} F\left(\cdot, \pi_{t+1}^{*}, z_{t+1}\right) Q_{t+1}\left(z_{0}, \ldots, z_{t} ; d z_{t+1}\right)
$$


over the set $\Gamma\left(\pi_{t-1}^{*}, z_{t}\right) \cap \operatorname{Proj}_{x} \Gamma^{-1}\left(\pi_{t+1}^{*}, z_{t+1}\right)$. The interpretation of (2) is similar of those of the deterministic case in the variational approach context: given an optimal (or at least finitely optimal) plan, any deviation from the optimal path at a certain date determines a lower value for the expected total return function.

Proof. Fix $s_{0} \in S, t \geq 1$ and consider a finitely optimal plan $\pi^{*} \in \Pi\left(s_{0}\right)$. Choose a feasible plan $\pi \in \Pi\left(s_{0}\right)$ with all elements equals a.e. to that of $\pi^{*}$ apart from the $t$-th; that is $\pi=\left(\pi_{0}^{*}, \pi_{1}^{*}, \ldots, \pi_{t-1}^{*}, y, \pi_{t+1}^{*}, \ldots\right)$. Since $\pi$ is feasible, $y \in \Gamma\left(\pi_{t-1}^{*}, z_{t}\right)$ and $\pi_{t+1}^{*} \in \Gamma\left(y, z_{t+1}\right)$. For all $n \geq t+1$, by the finite optimality condition, $u_{n}\left(s_{0}, \pi\right) \leq u_{n}\left(s_{0}, \pi^{*}\right)$ holds, which, using Corollary 1, can be written as follows,

$$
\begin{aligned}
& F\left(x_{0}, \pi_{0}^{*}, z_{0}\right)+\sum_{k=1}^{t-1} \beta^{k} \int_{Z^{k}} F\left(\pi_{k-1}^{*}, \pi_{k}^{*}, z_{k}\right) \lambda^{k}\left(z_{0}, d z^{k}\right) \\
& +\beta^{t} \int_{Z^{t}}\left[F\left(\pi_{t-1}^{*}, y, z_{t}\right)+\beta \int_{Z} F\left(y, \pi_{t+1}^{*}, z_{t+1}\right) Q_{t+1}\left(z_{0}, \ldots, z_{t} ; d z_{t+1}\right)\right] \lambda^{t}\left(z_{0}, d z^{t}\right) \\
& +\sum_{k=t+2}^{n} \beta^{k} \int_{Z^{k}} F\left(\pi_{k-1}^{*}, \pi_{k}^{*}, z_{k}\right) \lambda^{k}\left(z_{0}, d z^{k}\right) \\
& \leq F\left(x_{0}, \pi_{0}^{*}, z_{0}\right)+\sum_{k=1}^{t-1} \beta^{k} \int_{Z^{k}} F\left(\pi_{k-1}^{*}, \pi_{k}^{*}, z_{k}\right) \lambda^{k}\left(z_{0}, d z^{k}\right) \\
& +\beta^{t} \int_{Z^{t}}\left[F\left(\pi_{t-1}^{*}, \pi_{t}^{*}, z_{t}\right)+\beta \int_{Z} F\left(\pi_{t}^{*}, \pi_{t+1}^{*}, z_{t+1}\right) Q_{t+1}\left(z_{0}, \ldots, z_{t} ; d z_{t+1}\right)\right] \lambda^{t}\left(z_{0}, d z^{t}\right) \\
& +\sum_{k=t+2}^{n} \beta^{k} \int_{Z^{k}} F\left(\pi_{k-1}^{*}, \pi_{k}^{*}, z_{k}\right) \lambda^{k}\left(z_{0}, d z^{k}\right) .
\end{aligned}
$$

Simplifying equal terms in both members, we get (2).

Conditions (2) are very simple to check when the one-period return function is differentiable and differentiating under the integral is legitimate. Before restating (2) in the differentiable case, we obviously need a notion of interiority for the plan.

Definition 6 A feasible plan $\pi \in \Pi\left(s_{0}\right)$ is called interior plan if $\pi_{0} \in \operatorname{int} \Gamma\left(x_{0}, z_{0}\right)$ and $\pi_{t}\left(z^{t}\right) \in$ $\operatorname{int} \Gamma\left[\pi_{t-1}\left(z^{t-1}\right), z_{t}\right]$ a.e., $t=1,2, \ldots$.

A. $3 \operatorname{Int} X \neq \emptyset$ and For each $z \in Z, F(\cdot, \cdot, z): A_{z} \rightarrow \mathbf{R}$, that is the z-section of $F$, is differentiable on $\operatorname{int} A_{z}$ with each of the $l$ partial derivatives $F_{x}$ absolutely integrable.

This assumption permits the exchange of the differentiation and integration operators ${ }^{4}$ (see Lemma 2.2 , p. 226 in [5]); that is, for any probability measure $\lambda$ on $(Z, \mathcal{Z})$,

$$
D_{x}\left[\int F(x, y, z) \lambda(d z)\right]=\int F_{x}(x, y, z) \lambda(d z) .
$$

Proposition 4 Under Assumption 3, if a plan $\pi^{*} \in \Pi\left(s_{0}\right)$ is interior and optimal, or finitely optimal, or such that satisfies (2), then satisfies a.e.

$$
\begin{aligned}
& F_{y}\left(x_{0}, \pi_{0}^{*}, z_{0}\right)+\beta \int_{Z} F_{x}\left(\pi_{0}^{*}, \pi_{1}^{*}, z_{1}\right) Q_{1}\left(z_{0} ; d z_{1}\right)=0 \text { and } \\
& F_{y}\left(\pi_{t-1}^{*}, \pi_{t}^{*}, z_{t}\right)+\beta \int_{Z} F_{x}\left(\pi_{t}^{*}, \pi_{t+1}^{*}, z_{t+1}\right) Q_{t+1}\left(z_{0}, \ldots, z_{t} ; d z_{t+1}\right)=0, \\
& t=1,2, \ldots
\end{aligned}
$$

\footnotetext{
${ }^{4}$ Notice that any other condition that allows to differentiate under the integral sign can be taken into consideration as well.
} 
Conditions (3) are the stochastic version of the Euler-Lagrange equations in the deterministic case.

If the stochastic process of exogenous shocks is assumed to be a first order stationary Markov process characterized by a constant transition function $Q: Z \times \mathcal{Z} \rightarrow[0,1]$, the equations become the well known

$$
\begin{aligned}
& F_{y}\left(x_{0}, \pi_{0}^{*}, z_{0}\right)+\beta \int_{Z} F_{x}\left(\pi_{0}^{*}, \pi_{1}^{*}, z_{1}\right) Q\left(z_{0} ; d z_{1}\right)=0 \text { and } \\
& F_{y}\left(\pi_{t-1}^{*}, \pi_{t}^{*}, z_{t}\right)+\beta \int_{Z} F_{x}\left(\pi_{t}^{*}, \pi_{t+1}^{*}, z_{t+1}\right) Q\left(z_{t} ; d z_{t+1}\right)=0, \text { a.e., } t=1,2, \ldots
\end{aligned}
$$

Proof. Since optimality implies finite optimality, which in turn implies conditions (2), it is sufficient to show that conditions (2), plus interiority, imply (3). It follows from conditions (2) that, for each $t \geq 0$, the function $F\left(\pi_{t-1}^{*}, \cdot, z_{t}\right)+\beta \int_{Z} F\left(\cdot, \pi_{t+1}^{*}, z_{t+1}\right) Q_{t+1}\left(z_{0}, \ldots, z_{t} ; d z_{t+1}\right)$ reaches its maximum on $\pi_{t}^{*}$, which is an interior point of $\Gamma\left(\pi_{t-1}^{*}, z_{t}\right) \cap \operatorname{Proj}_{x} \Gamma^{-1}\left(\pi_{t+1}^{*}, z_{t+1}\right)$. By Assumption 3 that function is differentiable, hence (3) is the first order condition.

To complete this section we show that adding convexity of the set $A_{z}$ and concavity of $F(\cdot, \cdot, z): A_{z} \rightarrow \mathbf{R}$ for each $z \in Z$, Euler-Lagrange conditions become also sufficient for finite optimality.

Theorem 1 Assume that, for all $z \in Z, A_{z}$ is convex and $F(\cdot, \cdot, z): A_{z} \rightarrow \mathbf{R}$ is concave; in addition $F$ satisfies Assumption 3. Then, for each $s_{0} \in S$, a feasible plan $\pi^{*} \in \Pi\left(s_{0}\right)$ is finitely optimal if and only if is interior and satisfies conditions (3).

Proof. We have to prove only sufficiency. Fix $s_{0} \in S$ and $n \geq 1$. Choose two plans, $\pi$ and $\pi^{*}$ in $\Pi\left(s_{0}\right)$ such that $\pi^{*}$ is interior and satisfies (3) and $\pi$ is such that $\pi_{n}=\pi_{n}^{*}$ a.e.. Call $H$ the difference between the $n$-step return function $u_{n}\left(s_{0}, \cdot\right)$ evaluated at $\pi^{*}$ and at $\pi$, that is $H=\left[F\left(x_{0}, \pi_{0}^{*}, z_{0}\right)-F\left(x_{0}, \pi_{0}, z_{0}\right)\right]+\sum_{t=1}^{n} \beta^{t} \int_{Z^{t}}\left[F\left(\pi_{t-1}^{*}, \pi_{t}^{*}, z_{t}\right)-F\left(\pi_{t-1}, \pi_{t}, z_{t}\right)\right] \lambda^{t}\left(z_{0}, d z^{t}\right)$. Since $n$ is arbitrary, it is sufficient to show that $H$ is non-negative. It is well known that concavity is preserved under integration (see, e.g., Lemma 9.5, p.261 in [14]); hence, by concavity and differentiability of $F$,

$$
\begin{aligned}
H \geq & F_{x}\left(x_{0}, \pi_{0}^{*}, z_{0}\right)\left(x_{0}-x_{0}\right)+F_{y}\left(x_{0}, \pi_{0}^{*}, z_{0}\right)\left(\pi_{0}^{*}-\pi_{0}\right) \\
& +\sum_{t=1}^{n} \beta^{t} \int_{Z^{t}}\left[F_{x}\left(\pi_{t-1}^{*}, \pi_{t}^{*}, z_{t}\right)\left(\pi_{t-1}^{*}-\pi_{t-1}\right)+F_{y}\left(\pi_{t-1}^{*}, \pi_{t}^{*}, z_{t}\right)\left(\pi_{t}^{*}-\pi_{t}\right)\right] \lambda^{t}\left(z_{0}, d z^{t}\right) \\
= & {\left[F_{y}\left(x_{0}, \pi_{0}^{*}, z_{0}\right)+\beta \int_{Z} F_{x}\left(\pi_{0}^{*}, \pi_{1}^{*}, z_{1}\right) Q_{1}\left(z_{0} ; d z_{1}\right)\right]\left(\pi_{0}^{*}-\pi_{0}\right) } \\
& +\sum_{t=1}^{n-1} \beta^{t} \int_{Z^{t}}\left[F_{y}\left(\pi_{t-1}^{*}, \pi_{t}^{*}, z_{t}\right)\right. \\
& \left.+\beta \int_{Z} F_{x}\left(\pi_{t}^{*}, \pi_{t+1}^{*}, z_{t+1}\right) Q_{t+1}\left(z_{0}, \ldots, z_{t} ; d z_{t+1}\right)\right]\left(\pi_{t}^{*}-\pi_{t}\right) \lambda^{t}\left(z_{0}, d z^{t}\right) \\
& +\beta^{n} \int_{Z^{n}} F_{y}\left(\pi_{n-1}^{*}, \pi_{n}^{*}, z_{n}\right)\left(\pi_{n}^{*}-\pi_{n}\right) \lambda^{n}\left(z_{0}, d z^{n}\right),
\end{aligned}
$$

where the last four lines are obtained rearranging terms in the summation of the first two lines and applying Corollary 1. By (3) and the fact that $\pi_{n}=\pi_{n}^{*}$ a.e., the terms in the last summation are all zero, then $H \geq 0$.

\section{Stochastic Competitive Plans}

In this section we drop differentiability and concavity assumptions on the one-period return function $F$ and present a simple method in order to identify an optimal plan. We will only implicitly assume that $F$ is bounded from above and is superdifferentiable on the subset of its 
domain where the values of the random variables $\pi_{t}^{*}$ of an optimal plan $\pi^{*}$ lay. To make our approach more general, we consider a new optimization problem, equivalent to the one studied in the previous sections, characterized by a one-period return function infinitely penalized outside its domain $A$. Define $U: \mathbf{R}^{l} \times \mathbf{R}^{l} \times \mathbf{R}^{k} \rightarrow \mathbf{R} \cup\{-\infty\}$ by

$$
U(x, y, z)=\left\{\begin{array}{l}
F(x, y, z,) \text { if }(x, y, z) \in A \\
-\infty \text { otherwise. }
\end{array}\right.
$$

In other words we transform a problem with constraints (described by the correspondence $\Gamma$ ) into an unconstrained problem. The advantage of this procedure is that optimal plans with values on the boundary can also be considered throughout our method when $U$ is superdifferentiable on the boundary of the set $A$.

Recall that a function $f: X \rightarrow \mathbf{R} \cup\{-\infty\}$ is superdifferentiable at $x_{0} \in X \subseteq \mathbf{R}^{l}$ if there is a vector $a \in \mathbf{R}^{l}$ such that $f(x) \leq f\left(x_{0}\right)+a\left(x-x_{0}\right)$, all $x \in X ; a$ is called supergradient at $x_{0}$ and the (possibly empty) set of supergradients of $f$ at $x_{0}$, called superdifferential, is denoted by $\partial f\left(x_{0}\right)$.

For each $t \geq 1$, define the operator $M_{t}$ on the space of measurable functions $f: Z^{t} \rightarrow \mathbf{R}^{l}$ by

$$
\left(M_{t} f\right)\left(z^{t-1}\right)=\int_{Z} f\left(z^{t-1}, z_{t}\right) Q_{t}\left(z_{0}, \ldots, z_{t-1} ; d z_{t}\right) .
$$

It represents the expected value of the $t$-section of $f$ conditioned to the story of shocks $z^{t-1}=$ $\left(z_{1}, \ldots, z_{t-1}\right)$.

Definition 7 A feasible plan $\pi^{*} \in \Pi\left(s_{0}\right)$ is supported by a price $p_{0} \in \mathbf{R}^{l}$ and a sequence of prices $\left\{p_{t}\right\}$, where each $p_{t}: Z^{t} \rightarrow \mathbf{R}^{l}$ is a measurable function, if satisfies a.e.

$$
\begin{aligned}
& -\beta p_{0} \in \partial_{2} U\left(x_{0}, \pi_{0}^{*}, z_{0}\right) \text { and } \\
& \left(p_{t}\left(z^{t}\right),-\beta\left(M_{t+1} p_{t+1}\right)\left(z^{t}\right)\right) \in \partial U\left[\pi_{t-1}^{*}\left(z^{t-1}\right), \pi_{t}^{*}\left(z^{t}\right), z_{t}\right], t=1,2, \ldots,
\end{aligned}
$$

where we denote by $\partial_{2} U(\cdot, \cdot, \cdot)$ the superdifferential of $U$ with respect to the second variable and by $\partial U(\cdot, \cdot, \cdot)$ the superdifferential of $U$ with respect to the first and the second variable.

This is the stochastic version of what in the deterministic literature (see in particular [3] and [15]) is called competitive plans. The major difference with respect to the deterministic definition (see Definition 12.3, p. 127 in [10]) is that in stochastic models prices are random variables: they are unknown at the initial date $t=0$ and each of them is revealed (it becomes a vector of $\mathbf{R}^{l}$ ) as soon as the story of shocks $z^{t}=\left(z_{1}, \ldots, z_{t}\right)$ is observed. It is a concept analogue to that of feasible plans of Definition 1. This explains why in (4), at each period $t$, the price at $t+1$ appears in terms of expected value of the random variable $p_{t+1}$ conditioned to the story $z^{t}$. Notice that $p_{t}\left(z^{t}\right),\left(M_{t+1} p_{t+1}\right)\left(z^{t}\right)$ and $U\left[\pi_{t-1}^{*}\left(z^{t-1}\right), \pi_{t}^{*}\left(z^{t}\right), z_{t}\right]$ are all functions evaluated at time $t$, using information available at that period.

If the random shocks are represented by a first order stationary Markov process, as it is assumed in most of the economic models, operator $M_{t+1}$ in (4) becomes the well known Markov operator $M$ associated with the stationary transition function $Q$, i.e. $(M f)(z)=$ $\int_{Z} f\left(z^{\prime}\right) Q\left(z ; d z^{\prime}\right)$. Hence $\left(M p_{t+1}\right)\left(z^{t}\right)=\int_{Z} p_{t+1}\left(z^{t}, z_{t+1}\right) Q\left(z_{t} ; d z_{t}\right)$.

In applying the definition of superdifferentiability we can rewrite (4) as follows (again we omit the argument $z^{t}$ of the random variables $\pi_{t}, p_{t}$ and $\left.M_{t+1} p_{t+1}\right)$ :

$$
\begin{aligned}
& U\left(x_{0}, \pi_{0}^{*}, z_{0}\right)+\beta p_{0} \pi_{0}^{*} \geq U\left(x_{0}, \pi_{0}, z_{0}\right)+\beta p_{0} \pi_{0} \text { and } \\
& U\left(\pi_{t-1}^{*}, \pi_{t}^{*}, z_{t}\right)-p_{t} \pi_{t-1}^{*}+\beta\left(M_{t+1} p_{t+1}\right) \pi_{t}^{*} \\
& \quad \geq U\left(\pi_{t-1}, \pi_{t}, z_{t}\right)-p_{t} \pi_{t-1}+\beta\left(M_{t+1} p_{t+1}\right) \pi_{t} \text { a.e., all } \pi \in \Pi\left(s_{0}\right), \\
& t=1,2, \ldots
\end{aligned}
$$


It is easy to see that (5) are a generalization of (3): if $\pi^{*}$ is interior and $U$ satisfies Assumption 3 , we have $U_{x}\left[\pi_{t}^{*}\left(z^{t}\right), \pi_{t+1}^{*}\left(z^{t+1}\right), z_{t+1}\right]=p_{t+1}\left(z^{t+1}\right)$ and

$$
\begin{aligned}
U_{y}\left[\pi_{t-1}^{*}\left(z^{t-1}\right), \pi_{t}^{*}\left(z^{t}\right), z_{t}\right] & =-\beta\left(M_{t+1} p_{t+1}\right)\left(z^{t}\right) \\
& =-\beta \int_{Z} p_{t+1}\left(z^{t}, z_{t+1}\right) Q_{t+1}\left(z_{0}, \ldots, z_{t} ; d z_{t+1}\right) \\
& =-\beta \int_{Z} U_{x}\left[\pi_{t}^{*}\left(z^{t}\right), \pi_{t+1}^{*}\left(z^{t+1}\right), z_{t+1}\right] Q_{t+1}\left(z_{0}, \ldots, z_{t} ; d z_{t+1}\right),
\end{aligned}
$$

from which follows

$$
U_{y}\left[\pi_{t-1}^{*}\left(z^{t-1}\right), \pi_{t}^{*}\left(z^{t}\right), z_{t}\right]+\beta \int_{Z} U_{x}\left[\pi_{t}^{*}\left(z^{t}\right), \pi_{t+1}^{*}\left(z^{t+1}\right), z_{t+1}\right] Q_{t+1}\left(z_{0}, \ldots, z_{t} ; d z_{t+1}\right)=0 .
$$

Thus under differentiability the price system is uniquely determined.

Now we are ready to prove the main result.

Theorem 2 If a feasible plan $\pi^{*} \in \Pi\left(s_{0}\right)$ is supported by a sequence of random prices $\left\{p_{t}\right\}$ (i.e. is a competitive plan), then is finitely optimal. If, in addition, one of the following transversality conditions is satisfied, then $\pi^{*}$ is optimal:

1. $X$ is bounded and $\lim _{t \rightarrow \infty} \beta^{t} \int_{Z^{t}} p_{t}\left(z^{t}\right) \lambda^{t}\left(z_{0}, d z^{t}\right)=0$ a.e.;

2. $X \subset \mathbf{R}_{+}^{l}, p_{0} \in \mathbf{R}_{+}^{l}, p_{t}\left(z^{t}\right) \in \mathbf{R}_{+}^{l}$ a.e., $t=1,2, \ldots$ and $\lim _{t \rightarrow \infty} \beta^{t} \int_{Z^{t}} p_{t}\left(z^{t}\right) \pi_{t-1}^{*}\left(z^{t-1}\right) \lambda^{t}\left(z_{0}, d z^{t}\right)=$ 0 a.e.;

3. $\limsup _{t \rightarrow \infty} \beta^{t} \int_{Z^{t}} p_{t}\left(z^{t}\right)\left[\pi_{t-1}^{*}\left(z^{t-1}\right)-\pi_{t-1}\left(z^{t-1}\right)\right] \lambda^{t}\left(z_{0}, d z^{t}\right) \geq 0$ a.e., all $\pi \in \Pi\left(s_{0}\right)$.

Proof. We start with finite optimality. Fix $n \geq 1$ and choose any feasible plan $\pi \in \Pi\left(s_{0}\right)$ such that $\pi_{n}=\pi_{n}^{*}$ a.e.. By $(5)$, for $t=0,1, \ldots, n$,

$$
\begin{aligned}
& U\left[\pi_{t-1}^{*}\left(z^{t-1}\right), \pi_{t}^{*}\left(z^{t}\right), z_{t}\right]-p_{t}\left(z^{t}\right) \pi_{t-1}^{*}\left(z^{t-1}\right)+\beta\left(M_{t+1} p_{t+1}\right)\left(z^{t}\right) \pi_{t}^{*}\left(z^{t}\right) \\
& \quad \geq U\left[\pi_{t-1}\left(z^{t-1}\right), \pi_{t}\left(z^{t}\right), z_{t}\right]-p_{t}\left(z^{t}\right) \pi_{t-1}\left(z^{t-1}\right)+\beta\left(M_{t+1} p_{t+1}\right)\left(z^{t}\right) \pi_{t}\left(z^{t}\right),
\end{aligned}
$$

holds a.e., where the case with $t=0$ is also included since $x_{0}$ is in common to both $\pi$ and $\pi^{*}$ and, by the first line in (5), $U\left(x_{0}, \pi_{0}^{*}, z_{0}\right)-p x_{0}+\beta p_{0} \pi_{0}^{*} \geq U\left(x_{0}, \pi_{0}, z_{0}\right)-p x_{0}+\beta p_{0} \pi_{0}$ holds for any $p \in \mathbf{R}^{l}$. Then, writing explicitly $M_{t+1} p_{t+1}$, integrating with respect to $\bar{\lambda}^{t}\left(z_{0}, \cdot\right)$ and multiplying $\bar{b} y \beta^{t}$, we obtain

$$
\begin{aligned}
& \beta^{t} \int_{Z^{t}}\left\{U\left[\pi_{t-1}^{*}\left(z^{t-1}\right), \pi_{t}^{*}\left(z^{t}\right), z_{t}\right]-p_{t}\left(z^{t}\right) \pi_{t-1}^{*}\left(z^{t-1}\right)\right. \\
& \left.\quad+\beta \pi_{t}^{*}\left(z^{t}\right) \int_{Z} p_{t+1}\left(z^{t+1}\right) Q_{t+1}\left(z_{0}, \ldots, z_{t} ; d z_{t+1}\right)\right\} \lambda^{t}\left(z_{0}, d z^{t}\right) \\
& \geq \beta^{t} \int_{Z^{t}}\left\{U\left[\pi_{t-1}\left(z^{t-1}\right), \pi_{t}\left(z^{t}\right), z_{t}\right]-p_{t}\left(z^{t}\right) \pi_{t-1}\left(z^{t-1}\right)\right. \\
& \left.\quad+\beta \pi_{t}\left(z^{t}\right) \int_{Z} p_{t+1}\left(z^{t+1}\right) Q_{t+1}\left(z_{0}, \ldots, z_{t} ; d z_{t+1}\right)\right\} \lambda^{t}\left(z_{0}, d z^{t}\right)
\end{aligned}
$$

which, by Corollary 1, is equal to

$$
\begin{aligned}
& \quad \beta^{t} \int_{Z^{t}} U\left[\pi_{t-1}^{*}\left(z^{t-1}\right), \pi_{t}^{*}\left(z^{t}\right), z_{t}\right] \lambda^{t}\left(z_{0}, d z^{t}\right)-\beta^{t} \int_{Z^{t}} p_{t}\left(z^{t}\right) \pi_{t-1}^{*}\left(z^{t-1}\right) \lambda^{t}\left(z_{0}, d z^{t}\right) \\
& \quad+\beta^{t+1} \int_{Z^{t+1}} p_{t+1}\left(z^{t+1}\right) \pi_{t}^{*}\left(z^{t}\right) \lambda^{t+1}\left(z_{0}, d z^{t+1}\right) \\
& \geq \beta^{t} \int_{Z^{t}} U\left[\pi_{t-1}\left(z^{t-1}\right), \pi_{t}\left(z^{t}\right), z_{t}\right] \lambda^{t}\left(z_{0}, d z^{t}\right)-\beta^{t} \int_{Z^{t}} p_{t}\left(z^{t}\right) \pi_{t-1}\left(z^{t-1}\right) \lambda^{t}\left(z_{0}, d z^{t}\right) \\
& \quad+\beta^{t+1} \int_{Z^{t+1}} p_{t+1}\left(z^{t+1}\right) \pi_{t}\left(z^{t}\right) \lambda^{t+1}\left(z_{0}, d z^{t+1}\right) .
\end{aligned}
$$


Finally, the sum of both members from $t=0$ to $t=n$ gives

$$
\begin{aligned}
& \sum_{t=0}^{n} \beta^{t} \int_{Z^{t}} U\left[\pi_{t-1}^{*}\left(z^{t-1}\right), \pi_{t}^{*}\left(z^{t}\right), z_{t}\right] \lambda^{t}\left(z_{0}, d z^{t}\right) \\
& \quad+\beta^{n+1} \int_{Z^{n+1}} p_{n+1}\left(z^{n+1}\right) \pi_{n}^{*}\left(z^{n}\right) \lambda^{n+1}\left(z_{0}, d z^{n+1}\right) \\
& \geq \sum_{t=0}^{n} \beta^{t} \int_{Z^{t}} U\left[\pi_{t-1}\left(z^{t-1}\right), \pi_{t}\left(z^{t}\right), z_{t}\right] \lambda^{t}\left(z_{0}, d z^{t}\right) \\
& \quad+\beta^{n+1} \int_{Z^{n+1}} p_{n+1}\left(z^{n+1}\right) \pi_{n}\left(z^{n}\right) \lambda^{n+1}\left(z_{0}, d z^{n+1}\right),
\end{aligned}
$$

which, since $\pi_{n}=\pi_{n}^{*}$ a.e., implies finite optimality of $\pi^{*}$.

The final step is to prove optimality. Inequality (6) can be rewritten as follows:

$$
\begin{aligned}
& u_{n}\left(s_{0}, \pi^{*}\right)+\beta^{n+1} \int_{Z^{n+1}} p_{n+1}\left(z^{n+1}\right) \pi_{n}^{*}\left(z^{n}\right) \lambda^{n+1}\left(z_{0}, d z^{n+1}\right) \\
& \geq u_{n}\left(s_{0}, \pi\right)+\beta^{n+1} \int_{Z^{n+1}} p_{n+1}\left(z^{n+1}\right) \pi_{n}\left(z^{n}\right) \lambda^{n+1}\left(z_{0}, d z^{n+1}\right) .
\end{aligned}
$$

Suppose that condition 1 is verified; hence, writing (6) as

$$
u_{n}\left(s_{0}, \pi^{*}\right) \geq u_{n}\left(s_{0}, \pi\right)+\beta^{n+1} \int_{Z^{n+1}} p_{n+1}\left(z^{n+1}\right)\left[\pi_{n}\left(z^{n}\right)-\pi_{n}^{*}\left(z^{n}\right)\right] \lambda^{n+1}\left(z_{0}, d z^{n+1}\right)
$$

and taking the limit for $n \rightarrow \infty$, since $X$ is bounded, $u\left(s_{0}, \pi^{*}\right) \geq u\left(s_{0}, \pi\right)$ holds. If condition 2 is satisfied, then $p_{n+1}\left(z^{n+1}\right) \pi_{n}\left(z^{n}\right) \geq 0$ a.e. for $n=1,2, \ldots$, from which follows that $u_{n}\left(s_{0}, \pi^{*}\right)+$ $\beta^{n+1} \int_{Z^{n+1}} p_{n+1}\left(z^{n+1}\right) \pi_{n}^{*}\left(z^{n}\right) \lambda^{n+1}\left(z_{0}, d z^{n+1}\right) \geq u_{n}\left(s_{0}, \pi\right)$ holds for $n=1,2, \ldots$, and thus, again by condition 2 , taking the limit for $n \rightarrow \infty, u\left(s_{0}, \pi^{*}\right) \geq u\left(s_{0}, \pi\right)$ holds once more. The case of condition 3 is treated similarly.

\section{An Example}

Consider the one sector growth model with production possibilities affected by a stochastic exogenous shock. The objective of the decision maker in each period is to select consumption and investment policies to maximize expected discounted utility of consumption subject to an output generated by a stochastic production function.

The state space is $X=\mathbf{R}_{+}$, where $x_{t} \in X$ is the level of capital to be used in the production process at the beginning of period $t$; the output produced can be either consumed or invested. We assume that the investment choice $y_{t} \in X$ at time $t$ is equal to the amount of capital $x_{t+1}$ available at the beginning of the next period. The random events space is the closed interval $Z=[a, b] \subset \mathbf{R}$, where $0<a<b$, and we assume that any shock $z \in Z$ enters multiplicatively a Cobb-Douglas production function $f(x)=x^{\alpha}, 0<\alpha<1$, which already takes into account depreciation; i.e., if $y_{t-1}=x_{t} \in X$ represents the investment choice at $t-1, z_{t} x_{t}^{\alpha}$ is the output available at the same period. Hence, the correspondence of the one-period constraint is the closed interval $\Gamma(x, z)=\left[0, z x^{\alpha}\right]$. The utility of the representative decision maker is $U(c)=\ln c$. Since, thanks to monotonicity of both production and utility functions, $y_{t}=z_{t} x_{t}^{\alpha}-c_{t}, t=0,1, \ldots$ (that is no capital is wasted), the one-period return function is $F(x, y, z)=\ln \left(z x^{\alpha}-y\right)$. Formally, the agent must seek a sequence $\left\{x_{t}\right\}$ to maximize $E\left[\sum_{t=0}^{\infty} \beta^{t} \ln \left(z_{t} x_{t}^{\alpha}-x_{t+1}\right)\right]$, where $0<\beta<1$. Notice that, under these assumptions, we can represent the space of the system as the compact set $S=[0, b] \times[a, b]$, since for any $x>b$, production is not sustainable.

The process of the random shocks is characterized by a sequence $\left\{Q_{t}\right\}$ of stochastic kernels; that is, the probability measure on it is as general as possible. Thus, for fixed $s_{0} \in S$, we can restate the problem with the symbols introduced in the previous sections:

$$
\sup _{\pi \in \Pi\left(s_{0}\right)}\left\{\ln \left(z_{0} x_{0}^{\alpha}-\pi_{0}\right)+\liminf _{n \rightarrow \infty} \sum_{t=1}^{n} \beta^{t} \int_{Z^{t}} \ln \left[z_{t}\left(\pi_{t-1}\left(z^{t-1}\right)\right)^{\alpha}-\pi_{t}\left(z^{t}\right)\right] \lambda^{t}\left(z_{0}, d z^{t}\right)\right\},
$$


where $\lambda^{t}\left(z_{0}, \cdot\right)$ is defined in (1).

By using the method developed in Section 4, we shall show that the feasible plan generated by the function $g(x, z)=\alpha \beta z x^{\alpha}$, i.e. the stationary (or Markov) plan $\pi$ in which the random variables $\pi_{t}: Z^{t} \rightarrow X$ are defined by $\pi_{t}\left(z^{t}\right)=\alpha \beta z_{t}\left[\pi_{t-1}\left(z^{t-1}\right)\right]^{\alpha}, t=1,2, \ldots$, is optimal. By using well known dynamic programming arguments, it is easy to show that the plan constructed in this way is optimal under standard hypotheses on the random shocks (like assuming it being an i.i.d. process or, at most, a first order Markov process); it seems interesting the fact that this plan remains optimal under much weaker assumptions on the uncertainty as well.

Clearly $\pi$ is feasible for any $s_{0} \in S$. Hence we must prove that $\pi$ is competitive and satisfy one of the transversality conditions of Theorem 2. The function $F(x, y, z)=\ln \left(z x^{\alpha}-y\right)$ is continuously differentiable on $\operatorname{int} A_{z}$, thus satisfies Assumption 3. Therefore we need to show that $\pi$ is interior and conditions (3) hold, or, in other words, that a sequence $\left\{p_{t}\right\}$ of supporting prices exists and is uniquely determined by (3).

Suppose that $x_{0}>0$. Since $z_{t}>0, t=0,1, \ldots$, then, by induction, $\alpha \beta z_{t} x_{t}^{\alpha}>0$, all $t$; furthermore $\alpha \beta z x^{\alpha}<z x^{\alpha}$, all $(x, z) \in S$, hence $\pi$ is interior. To check (3), we must show that

$$
\frac{1}{z_{t}\left[\pi_{t-1}\left(z^{t-1}\right)\right]^{\alpha}-\pi_{t}\left(z^{t}\right)}=\beta \int_{Z} \frac{\alpha z_{t+1}\left[\pi_{t}\left(z^{t}\right)\right]^{\alpha-1}}{z_{t+1}\left[\pi_{t}\left(z^{t}\right)\right]^{\alpha}-\pi_{t+1}\left(z^{t+1}\right)} Q_{t+1}\left(z_{0}, \ldots, z_{t} ; d z_{t+1}\right)
$$

holds a.e., $t=0,1, \ldots$ Using function $g$, we can substitute $\pi_{t-1}\left(z^{t-1}\right)$ and $\pi_{t+1}\left(z^{t+1}\right)$ with their equivalent expressions in terms of $\pi_{t}\left(z^{t}\right)$; then simplifying, by the arbitrarity of $t$ and $z^{t}$ and since $\int Q_{t+1}\left(z_{0}, \ldots, z_{t} ; d z_{t+1}\right)=1$ for all $\left(z_{0}, \ldots, z_{t}\right)$, we get the identity.

Finally we turn to the transversality condition. At each time $t$, the supporting price is

$$
p_{t}\left(z^{t}\right)=\frac{\alpha z_{t}\left[\pi_{t-1}\left(z^{t-1}\right)\right]^{\alpha-1}}{z_{t}\left[\pi_{t-1}\left(z^{t-1}\right)\right]^{\alpha}-\pi_{t}\left(z^{t}\right)}
$$

where, substituting $\pi_{t}\left(z^{t}\right)$ with $\alpha \beta z_{t}\left[\pi_{t-1}\left(z^{t-1}\right)\right]^{\alpha}$, we have $p_{t}\left(z^{t}\right)=\alpha /(1-\alpha \beta) \pi_{t-1}\left(z^{t-1}\right)$. Since, for all $t$ and $z^{t}, \pi_{t}\left(z^{t}\right)>0$, the price $p_{t}\left(z^{t}\right)$ is always strictly positive; in addition $X=\mathbf{R}_{+}$. To see that condition 2 of Theorem 2 is verified, notice that

$$
\begin{aligned}
\lim _{t \rightarrow \infty} \beta^{t} \int_{Z^{t}} p_{t}\left(z^{t}\right) \pi_{t-1}\left(z^{t-1}\right) \lambda^{t}\left(z_{0}, d z^{t}\right) & =\lim _{t \rightarrow \infty} \frac{\alpha \beta^{t}}{1-\alpha \beta} \int_{Z^{t}} \lambda^{t}\left(z_{0}, d z^{t}\right) \\
& =\frac{\alpha}{1-\alpha \beta} \lim _{t \rightarrow \infty} \beta^{t} \\
& =0,
\end{aligned}
$$

hence $\pi$ is optimal.

With a similar argument, it is easy to show that for an analogue problem with finite horizon $T$ and a final condition equal to zero, that is

$$
\begin{aligned}
\sup _{\pi \in \Pi\left(s_{0}\right)}\{ & \ln \left(z_{0} x_{0}^{\alpha}-\pi_{0}\right)+\sum_{t=1}^{T} \beta^{t} \int_{Z^{t}} \ln \left[z_{t}\left(\pi_{t-1}\left(z^{t-1}\right)\right)^{\alpha}-\pi_{t}\left(z^{t}\right)\right] \lambda^{t}\left(z_{0}, d z^{t}\right) \\
& \left.+\beta^{T} \int_{Z^{T}} \ln z_{T}\left[\pi_{T}\left(z^{T}\right)\right]^{\alpha} \lambda^{T}\left(z_{0}, d z^{T}\right)\right\},
\end{aligned}
$$

a plan constructed by the functions

$$
\pi_{t}\left(z^{t}\right)=\alpha \beta \frac{1-(\alpha \beta)^{T-t}}{1-(\alpha \beta)^{T-t+1}} z_{t}\left[\pi_{t-1}\left(z^{t-1}\right)\right]^{\alpha}
$$

is optimal.

The interpretation of such an apparently surprising result relies entirely upon the fact that logarithm is a well behaved function. The plan generated by the function $g(x, z)=$ 
$\alpha \beta z x$, in fact, is optimal also for the analogous deterministic model characterized by a nonstationary one-period return function, as can be easily checked; i.e. for a model of the type $\sup \sum_{t=0}^{\infty} \beta^{t} \ln \left(z_{t} x_{t}^{\alpha}-x_{t+1}\right)$, where $\left\{z_{t}\right\}$ is a deterministic sequence of numbers which let the one-period return function $U_{t}(c)=\ln \left(z_{t} x_{t}^{\alpha}-x_{t+1}\right)$ depend on time $t$. This means that in stochastic models logarithm utilities nullify the effects of uncertainty (of any kind), a circumstance that in general, obviously, doesn't occur.

\section{Concluding Remarks}

Although the majority of economic optimization models are not so fortunate as in the last section, where utility is the logarithm function, we believe that stochastic competitive plans, i.e. feasible plans supported by sequences of random prices, can be useful in determining optimal plans in many economic applications under uncertainty, particularly when the oneperiod return function is differentiable, assumption that allows to check competitivity by EulerLagrange equations (3). Equilibrium theory seems to be the field where competitive plans can play a crucial role: at any time $t$, an equilibrium is defined by a system of random prices $p_{t}$ (which depend on an exogenous shock $z_{t}$ ) and a system of demand and supply functions that maximize utility and clear the markets. It's easy to see that such an equilibrium corresponds to a competitive plan. Other important aspects deserve further investigation, like Pareto optimality of the equilibrium which involve the analysis of transversality conditions similar to that listed in Theorem 2.

\section{References}

[1] Blume, L., D. Easley and M. O'Hara, Characterization of Optimal Plans for Stochastic Dynamic Programs, Journal of Economic Theory 28, 1982: 221-234.

[2] Brock, W. A. and M. Majumdar, Global Asymptotic Stability Results for Multisector Models of Optimal Growth under Uncertainty When Future Utilities are Discounted, Journal of Economic Theory 18, 1978: 225-243.

[3] Gale, D., On Optimal Development in a Multi-Sector Economy, Review of Economic Studies 34, 1967: 1-18.

[4] Hildenbrand, W., Core and Equilibria of a Large Economy, Princeton, N. J.: Princeton University Press, 1974.

[5] Lang, S., Real and Functional Analysis, third edition, Springer-Verlag, 1993.

[6] Levhari, D. and T. N. Srinivasan, Optimal Savings under Uncertainty, Review of Economic Studies 36, 1969: 153-163.

[7] Majumdar, M. and I. Zilcha, Optimal Growth in a Stochastic Environment: Some Sensitivity and Turnpike Results, Journal of Economic Theory 43, 1987: 116-133.

[8] McKenzie, L. W., Optimal Economic Growth, Turnpike Theorems and Comparative Dynamics, in Handbooks in Economics III, Ed. by K. J. Arrow and M. D. Intriligator, NorthHolland, Amsterdam, 1986: 1281-1355. 
[9] Mirman, L. J. and I. Zilcha, Unbounded Shadow Prices for Optimal Stochastic Growth Models, International Economic Review 17, 1976: 121-132.

[10] Montrucchio, L., Introduzione all'Ottimizzazione Dinamica, University of Turin, Italy, 1994.

[11] Neveu, J., Mathematical Foundations of the Calculus of Probability, Trans. A. Fenstein, San Francisco: Holden Day, 1965.

[12] Radner, R., Optimal Stationary Consumption with Stochastic Production and Resources, Journal of Economic Theory 6, 1973: 68-90.

[13] Takekuma, S. I., Optimal Growth under Uncertainty: a Complete Characterization of Weakly Maximal Programs, Hitotsubashi Journal of Economics 33, 1992: 169-182.

[14] Stokey, N. L. and R. E. Lucas, Recursive Methods in Economic Dynamics, Cambridge, Mass.: Harvard University Press, 1989.

[15] Weitzman, M., Duality Theory for Infinite Horizon Convex Models, Management Science 19, 1973: 783-789.

[16] Yano, M., Comparative Statics in Dynamic Stochastic Models Journal of Mathematical Economics 18, 1989: 169-185.

[17] Zilcha, I., Characterization by Prices of Optimal Programs under Uncertainty, Journal of Mathematical Economics 3, 1976: 173-183. 